patients were exposed to acetarsol more than once. $76.7 \%$ of patients achieved clinical response. $3 / 35$ patients had an endoscopic assessment with two of three patients showing endoscopic improvement. $33.3 \%$ patients required treatment escalation following acetarsol exposure with two undergoing subtotal colectomy. Five patients (14.3\%) stopped acetarsol due to side effects. One patient experienced vomiting, palpitations and sweating, and the other four experienced headache, vomiting, anal itching and paresthesia. Median serum arsenic level was $728.25(872) \mathrm{nmol} / \mathrm{l}(<130 \mathrm{nmol} / \mathrm{L})$. Serum arsenic levels were not correlated with patient clinical response nor the need for treatment escalation.

Conclusions Acetarsol suppositories could be an effective and tolerable option in the management of refractory proctitis. A definitive study is urgently warranted to thoroughly investigate the clinical efficacy and safety of this promising drug.

\section{PWE-004 TAPERING WITH BUDESONIDE - LABEL RECOMMENDATION VERSUS CLINICAL REALITY}

${ }^{1}$ Philip Becker*, ${ }^{1}$ Robert Hofmann, ${ }^{2}$ Benny Chemnitz-Nielsen. ${ }^{1}$ Tillotts Pharma; Medical Affairs, Rheinfelden, Switzerland; ${ }^{2}$ Kantar Sifo, Göteborg, Sweden

\subsection{6/gutjnl-2018-BSGAbstracts. 136}

Introduction Symptoms of steroid withdrawal may be avoided in most patients by tapering the dose over time when deciding to discontinue treatment. Patients exposed to steroid therapy, even if only for a short period of time are very likely to have diminished hypothalamic-pituitary-adrenal (HPA) axis function. Despite primarily local action, budesonide can also affect the HPA axis. Tapering of budesonide allows the adrenal glands time to return to their normal patterns of secretion. This is a precaution and highlighted in the Entocort* Summary of Product Characteristics recommendation where it is stated 'when treatment is to be discontinued, the dose should normally be reduced for the last 2 to 4 weeks of therapy'. The presented study was investigating tapering habits for budesonide prescriptions in Crohn's disease (CD) among gastroenterologists in Europe.

Methods An online survey** was conducted among a total sample of 161 gastroenterologists in 8 countries. The data collection took place from November to December 2016. The physicians in the sample received a link to an online questionnaire (local language) on a secure platform via email. The questionnaire on prescribing practises included the following questions with regards to the use of budesonide for the treatment of CD: (i) Do you normally recommend tapering dose in the last 2-4 weeks of budesonide treatment? (ii) Do you recommend tapering over 2, 3, or 4 weeks? (iii) Do you recommend tapering to either $6 \mathrm{mg}$ then $3 \mathrm{mg}$, straight to $3 \mathrm{mg}$ or to $6 \mathrm{mg}$ only?

Results Of the 161 gastroenterologists surveyed, the majority (81\%) tapered when prescribing budesonide; the countries where the response rate was $\geq 80 \%$ included Finland (100\%), Norway (88\%), Czech Republic (82\%), Denmark (82\%) and Spain (80\%). Most gastroenterologists tapered over a 4 week time period $(78 \%)$, with a small minority over other time schedules (2 or 3 weeks). The most commonly used tapering schedule was 9-6-3-0 mg (91\%), with only 5\% and $4 \%$ of gastroenterologists tapering using a $9-6-0 \mathrm{mg}$ and $9-3-0 \mathrm{mg}$ schedule, respectively.
Conclusions The majority of gastroenterologists surveyed are adhering to the budesonide label recommendation of tapering over a 2-4 week period when prescribing budesonide. The reasons behind $20 \%$ of prescribers deviating from the product labels needs further research to be addressed.

* The rights to Entocort, including the rights to the trademark, are owned by Tillotts Pharma AG except for the USA.

*Financial sponsorship was provided by Tillotts Pharma AG.

\section{PWE-005 VEDOLIZUMAB DOSE ESCALATION AS A WAY OF RECAPTURING RESPONSE IN PATIENTS WITH INFLAMMATORY BOWEL DISEASE}

'S Birdi ${ }^{*},{ }^{1,2} \mathrm{M}$ Sierra Morales, ${ }^{2} \mathrm{MA}$ Samaan, ${ }^{1} \mathrm{C}$ Brown-Clarke, ${ }^{1} \mathrm{~A}$ Stanton, 'S Ray, 'I Koumoutsos, 'J Mawdsley, 'S Anderson, 'Sandersdon, 'PM Irving. 'Guy's and St Thomas' Hospital NHS Trust,Department of Gastroenterology, London, UK; ${ }^{2}$ Hospital Universitario Ramón y Cajal, Madrid, Spain

\subsection{6/gutjnl-2018-BSGAbstracts. 137}

Introduction Increasing vedolizumab (VDZ) dosing frequency to recapture response has been shown to be effective in clinical trials but there is limited real-life data from the clinical practice. In this study we assessed whether VDZ dose escalation helped recapture response in a large cohort of patients in a tertiary referral IBD centre.

Methods A retrospective cohort study was performed by reviewing prospectively recorded clinical data for patients who received VDZ between November 2014 and October 2017. Patients who had sub-optimal response and had been escalated to 6 or 4 weekly infusions were identified. Data collected for demographics, previous biologic exposure, concomitant immunomodulators (IM), steroid use (SU), clinical disease activity for CD (HBI) and UC (SCCAI), and CRP levels at baseline, 12 and 24 weeks after dose escalation.

Of the total 139 patients on VDZ, $36(27 \%)$ had been escalated to Q4 (30) or Q6 (6), of whom 5 were further escalated to Q4 (72\% male, median age 44 , previous biologics exposure $81 \%$, 49\% concomitant IM and $16 \%$ SU at time of escalation). 18 patients had CD (50\%), 14 UC (39\%), and 4 (11\%) IBD-U which were included in the UC group for the purpose of analysis.

Duration of VDZ before and after dose escalation with a median of $7 \mathrm{~m}$ (ranges 0-22, 2-25 respectively). Currently $76 \%$ remain on VDZ after dose escalation (median $7 \mathrm{~m}$ after escalation).

Clinical response was defined as HBI or SCCAI reduction $>3$. Remission as $\mathrm{HBI}<5$ or SCCAI $<3$. Paired HBI, SCCAI, CRP values at baseline, week 12 and 24 were compared using Wilcoxon signed-rank test

Results Patients with CD had a median HBI of 4 (range 027), 4 (0-29) and $3(0-8)$, at baseline, 12 and 24 weeks.

In UC group, the median SCCAI was 6 (range 0-11); 4.5 (1-11), and 4 (0-10), at baseline, 12 and 24 weeks.

CRP for both groups at baseline was a median of 6 (123), $5(1-46)$ at w12, and $2(1-17)$ at w24.

HBI and SCCAI at baseline, 12 and 24 weeks after dose escalation

Statistically significant differences were noted in the UC group between SCCAI at baseline and after 24 weeks ( $p$ 0.01) and overall CRP at baseline and $24 \mathrm{w}$ (p 0.04). 
Of all patients with clinically active disease at baseline $(\mathrm{n}=20), 5$ achieved clinical response $(25 \%)$, an additional 4 achieved clinical remission (20\%).
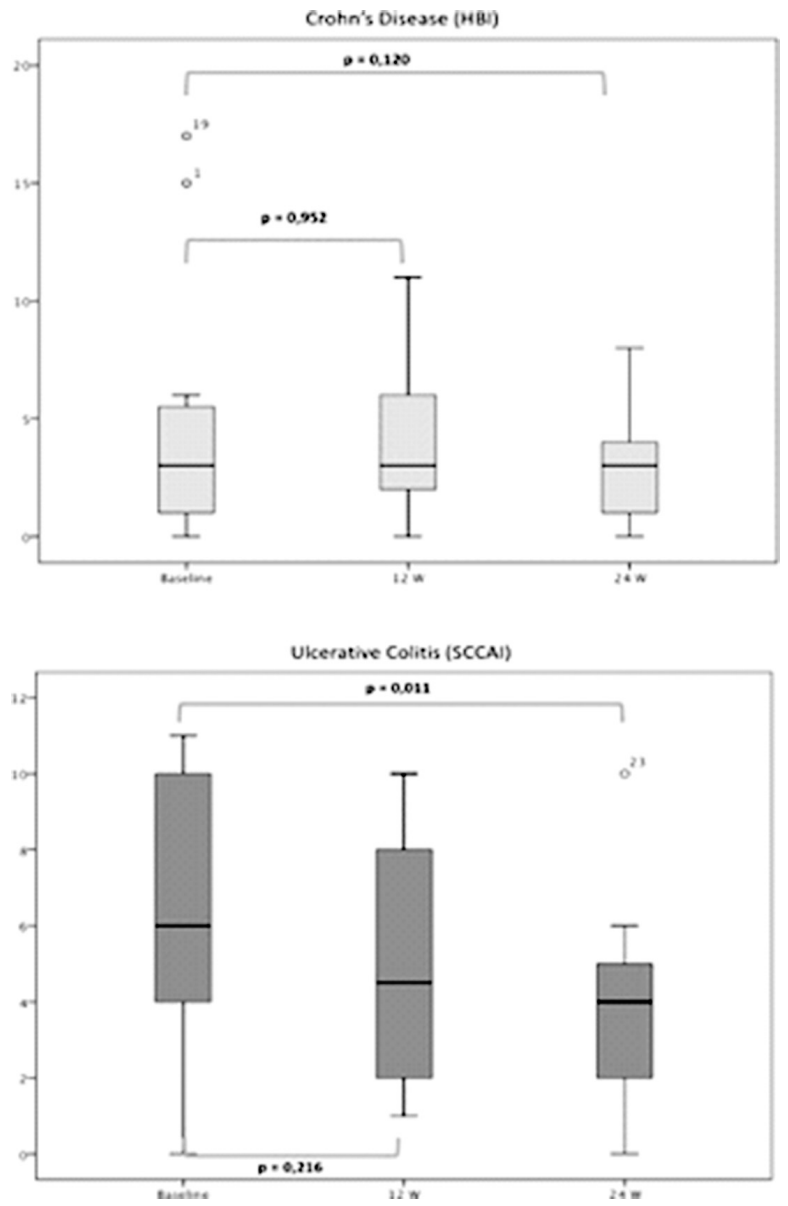

Abstract PWE-005 Figure 1

Conclusions In a real life setting, increasing dosing frequency in patients with sub-optimal response to VDZ is effective in approximately half of patients and should be considered as an intervention.

\section{PWE-006 SMOKING IN UC IS ASSOCIATED WITH DECREASED THIOPURINE USE BUT NOT STEROID DEPENDENCY OR COLECTOMY}

Jonathan *. St George's NHS Foundation Trust, South Croydon, UK

\subsection{6/gutjnl-2018-BSGAbstracts. 138}

Introduction Whilst smoking is established as a protective modifiable environmental risk factor for the development of Ulcerative Colitis (UC), the evidence for its impact on subsequent disease activity is conflicting. We therefore aimed to investigate the impact of smoking on clinical outcomes in the disease course of UC.

Methods Using a nationally representative clinical practice research database (CPRD), we identified incident cases of UC diagnosed between 2005 and 2014. Patients were grouped as: smokers, never-smokers or ex-smokers at UC diagnosis based on medical record codes for smoking status in the two years preceding UC diagnosis. Medical record codes were also examined to determine change in smoking status following diagnosis. We compared corticosteroid dependency (as defined in ECCO guidelines), thiopurine use and colectomy rates between these defined groups. Survival analysis, Cox proportional hazards analysis and logistic regression were used determine the risk of first thiopurine use, corticosteroid dependency and colectomy given smoking status.

Abstract PWE-006 Table 1 Univariate and multivariate Cox regression analysis for risk of Thiopurine use in patients with Ulcerative Colitis

\begin{tabular}{|c|c|c|c|c|c|c|}
\hline & \multicolumn{3}{|c|}{$\begin{array}{c}\text { Univariate analysis } \\
n=4058\end{array}$} & \multicolumn{3}{|c|}{$\begin{array}{c}\text { Multivariate analysis } \\
n=1,587\end{array}$} \\
\hline & HR & $95 \% \mathrm{Cl}$ & p value & HR & $95 \% \mathrm{CI}$ & p value \\
\hline \multicolumn{7}{|l|}{ Smoking status" } \\
\hline Never Smoker & 1 & - & - & - & - & - \\
\hline Persistent Smoker & 0.61 & $0.44-0.86$ & 0.005 & 0.52 & $0.27-0.97$ & 0.041 \\
\hline Persistent Ex-Smaker & 0.84 & $0.71 \cdot 0.99$ & 0.046 & 1.00 & $0.75-1.34$ & 0.95 \\
\hline New Smoker Post UC & 0.86 & $0.58-1.28$ & 0.485 & 0.84 & $0.43-1.62$ & 0.60 \\
\hline New Ex-Smoker Post UC & 0.96 & $0.69-1.33$ & 0.819 & 1.07 & $0.62-1.85$ & 0.80 \\
\hline Relapsed Smoker Post UC. & 1.23 & $0.90-1.68$ & 0.181 & 1.01 & $0.59-1.73$ & 0.94 \\
\hline \multicolumn{7}{|l|}{ Sex } \\
\hline Female & 1 & - & - & - & - & - \\
\hline Male & 1.26 & $1.08-1.45$ & 0.002 & 1.30 & $1.01-1.68$ & 0.040 \\
\hline \multicolumn{7}{|l|}{ Age at 180 diagnosis } \\
\hline A1 & 1 & - & - & $\therefore$ & $\therefore$ & - \\
\hline A2 & 0.53 & $0.31-0.89$ & 0.017 & 2.39 & $0.72-7.89$ & 0.15 \\
\hline $\mathrm{A} 3$ & 0.26 & $0.15-0.44$ & 0.000 & 1.11 & $0.33-3.65$ & 0.86 \\
\hline \multicolumn{7}{|l|}{ Era of 180 diagnosis } \\
\hline $2005 / 2006$ & 1 & - & - & - & - & - \\
\hline $2007 / 2008$ & 1.09 & $0.87-1.36$ & 0.429 & 0.92 & $0.63-1.35$ & 0.69 \\
\hline $2009 / 2010$ & 1.06 & $0.84-1.33$ & 0.593 & 1.10 & $0.76-1.59$ & 0.68 \\
\hline $2011 / 2012$ & 1.45 & $1.15-1.82$ & 0.001 & 1.49 & $1.02-2.18$ & 0.035 \\
\hline $2013 / 2014$ & 1.50 & 1.15-1.95 & 0.003 & 1.51 & $0.90-2.55$ & 0.11 \\
\hline \multicolumn{7}{|l|}{ BMI category' } \\
\hline Underweight & 0.96 & $0.57-1.63$ & 0.898 & 0.58 & $0.30-1.11$ & 0.10 \\
\hline Normal & 1 & - & - & - & - & - \\
\hline Overweight & 0.77 & $0.62-0.95$ & 0.016 & 0.89 & $0.67-1.19$ & 0.44 \\
\hline Obese & 0.72 & $0.56-0.92$ & 0.009 & 0.92 & $0.65-1.31$ & 0.66 \\
\hline \multicolumn{7}{|l|}{ Social deprivation" } \\
\hline IMO 1 & 1 & - & - & & - & - \\
\hline IMO 2 & 0.86 & $0.65-1.13$ & 0.306 & 0.87 & $0.61-1.24$ & 0.45 \\
\hline IMO 3 & 0.97 & $0.72-1.29$ & 0.838 & 0.95 & $0.64-1.40$ & 0.80 \\
\hline IMO 4 & 0.88 & $0.66-1.18$ & 0.412 & 1.01 & $0.70-1.46$ & 0.95 \\
\hline IMOS & 0.98 & $0.71-1.36$ & 0.940 & 0.83 & $0.56-1.24$ & 0.38 \\
\hline \multicolumn{7}{|l|}{ Co-morbidities' } \\
\hline IBS & 0.79 & $0.63-1.00$ & 0.055 & 0.60 & $0.40-0.88$ & 0.011 \\
\hline Depression & 0.75 & $0.57-0.99$ & 0.045 & 0.76 & $0.49-1.20$ & 0.24 \\
\hline \multicolumn{7}{|l|}{180 medications } \\
\hline Oral 5-ASA & 2.50 & $2.06-3.03$ & $<0.001$ & 1.57 & $1.16-2.13$ & 0.004 \\
\hline Corticosteroids & 8.57 & $6.86-10.71$ & $<0.001$ & $x$ & $\mathrm{x}$ & $\mathrm{x}$ \\
\hline \multicolumn{7}{|l|}{ Severity Indices" } \\
\hline Early Corticosteroid Use & 3.68 & $3.19-4.26$ & 0.008 & 1.43 & $1.09-1.87$ & 0.008 \\
\hline Colectomy & 3.20 & 2.49 .4 .12 & 0.007 & 1.76 & $1.16-2.67$ & 0.007 \\
\hline Corticosteroid Dependence & 5.62 & $4.85-6.51$ & $<0.001$ & 2.02 & $1.55-2.64$ & $<0.001$ \\
\hline \multicolumn{7}{|l|}{ Corticosteroid Flares Per Year } \\
\hline & 1 & - & - & & - & - \\
\hline $0-1$ & 8.39 & $6.69-10.51$ & $<0.001$ & 5.11 & $3.34-7.81$ & $<0.001$ \\
\hline$>1$ & 13.05 & $9.40-18.10$ & $<0.001$ & 6.15 & $3.22-11.72$ & $<0.001$ \\
\hline
\end{tabular}

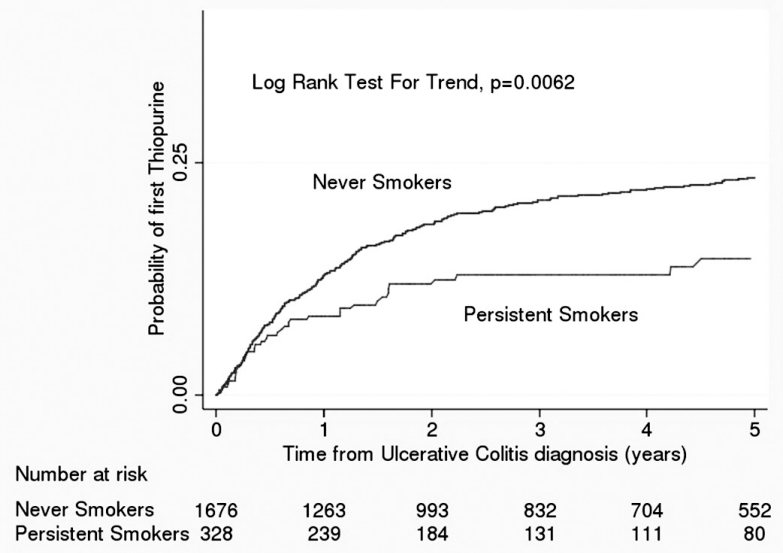

Abstract PWE-006 Figure 1 Kaplan Meier Curve: Progression to Thiopurine Use in Smokers and Never Smokers in Ulcerative Colitis 